\title{
Application of Electromagnetic Guns to Future Naval Platforms
}

\author{
W. A. Walls, W. F. Weldon, S. B. Pratap \\ Center for Electromechanics, The University of Texas at Austin \\ M. Palmer \\ Science Applications International Corporation \\ Lt. David Adams \\ U.S. Navy
}

\begin{abstract}
Designs for future naval vessels are strongly considering electric drive systems. Already employed in commercial cruise ships, electric drive offers the advantages of increased ship design flexibility, improved efficiency, reduced maintenance and allows ship prime power to be easily diverted to other electrical loads as needed. The ability to use ship prime power generation, which ranges between 40 and $150 \mathrm{MW}$ depending on vessel class, for other electric loads provides the opportunity to electrify many existing functions as well as add new performance enhancing systems. The recent and ongoing emergence of electric gun and guided projectile technologies now allows very long range naval fire support functions to be evaluated for viability. In this paper, conceptual system designs for surface fire support of forces in littoral campaigns are considered. Key advantages of an EM fire support weapon over conventional technologies include reduced logistics burden and cost per round, greater lethality, shorter time of flight, improved survivability, and the ability to stow more rounds. Notional mission requirements, projectile, power supply issues, and ship integration issues are discussed. Also, other shipboard uses for the pulse power system required for these notional electric gun systems are also reviewed.
\end{abstract}

\section{BACKGROUND}

Electromagnetic (EM) gun technologies have been under development by DARPA and the U.S. Army for about two decades. The promise of advantages including increased lethality, shorter time of flight, greater accuracy, reduced logistics, and improved survivability is the driving force for development of EM gun systems for land combat vehicles. During that development effort, substantial improvements in the relevant technologies have been achieved. Launchers have evolved from very heavy, low bore pressure devices

Manuscript received May 1, 1998.

W.A. Walls may be contacted at 512-471-4496, fax 512-471-0781.
[1][2] to lightweight composite reinforced structures capable of operating at bore pressures near that used in conventional powder guns [3][4][5]. Projectiles have evolved from small plastic cubes accelerated by plasma armatures [6][7] to fully capable launch packages with integral solid armature and sabot structures supporting relevant payload projectiles [8][9][10]. Compact power supplies using rotating machines to both store energy and generate the required current pulses for the gun are also being developed [11][12] to replace the large capacitor or iron-based rotating machine laboratory systems now in use. These previous development efforts have been primarily directed toward combat vehicle applications where weight and volume goals are very stringent. In most cases, the EML technology goals for the launcher, launch package, and power supply needed for combat vehicle application are now within reach. Furthermore, system integration studies [13] [14] suggest that prime power and thermal management burdens associated with electric guns are not prohibitive.

The U.S. Navy is currently developing gun launched, rocket-assisted guided projectiles for long range fire support applications. Global Positioning System-Inertial Navigation System (GPS/INS) guidance packages and aerodynamic surfaces are used to control the flight trajectory and impact point.

Given the current state of EM gun and guided projectile technologies, a new look at naval applications is now warranted. Naval combat vessels are perhaps the most suitable platform for initial fielding of EM guns. While mass and volume in naval ships is limited, it is much less restricted than in ground combat vehicles. A ship platform also has an abundant heat rejection medium in seawater, and a future ship using an integrated electric power system can easily provide adequate levels of prime power needed for sustained rates of fire. Both prime power and cooling availability are currently critical factors in defining operating limits for land vehicle mounted systems. 


\section{NAVAL FIRE SuPPORT MisSiON}

The most compelling application for EM guns in the future Navy is surface fire support. As the U.S. force structure is reduced in size and moved closer to home as forward bases are eliminated, the need for rapid response to regional crisis situations becomes more and more likely. In addition, the fact that $85 \%$ of the world's population resides within 200 miles of a shoreline [15] (littoral regions) indicates that many of these situations will erupt in locations where surface fire can be effective in supporting ground forces or disrupting threat forces. In order to provide adequate stand off from littoral threats to naval vessels and achieve sufficient cross-range engagement capability, a nominal range capability of 100 to $400 \mathrm{nmi}$ is of significant interest. Presently, fire support at this range is provided by tactical aircraft operating from carriers or by medium range guided missiles. Medium range guided missiles are not affordable for large volume fires, while naval aircraft delivery puts aircrews at risk, introducing numerous complex national policy issues. Aircraft also require relatively long times to fly to their targets, and have daily ordnance delivery rates limited by the number of available aircraft carriers.

EM guns can potentially launch very large numbers of guided weapons to medium ranges at very low cost. This can effectively multiply the fire power, mission effectiveness, and cost effectiveness of Naval task forces many fold. Naval aircraft would no longer be required to function as mere cargo carrying trucks, relieving them to more effectively accomplish missions where a manned aircraft is essential. This includes functions such as air defense, target verification, patrol, airspace command and control, and implementation of national policy.

Electromagnetic guns can reduce the cost of ordnance delivery and decrease response times, by over an order of magnitude in both cases. This technology can also increase by 10 to 100 fold the lethality which can be delivered per unit of underdeck stowed volume. This potentially can be accomplished using kinetic energy warhead technology which totally eliminates all reactive materials such as explosive or rocket propellants. This then eliminates major logistical costs and environmental and safety hazards associated with such materials.

Due to the increased velocity capability of the railgun, it is possible to use kinetic energy directly as the lethality mechanism rather than using chemical explosives to impart kinetic energy to fragments. This allows the use of high density materials like tungsten for the flight body, which reduces its size, and therefore its aerodynamic drag, considerably. Matching the total kinetic energy of the fragments created by a single $155 \mathrm{~mm}$ submunition cargo shell may require about 1 $\mathrm{kg}$ of material impacting at $2 \mathrm{~km} / \mathrm{s}$. The key is to provide sufficient residual velocity at impact so that explosives are not required. Fig. 1 shows how impact velocity varies with initial launch velocity and projectile mass. The velocities shown in Fig. 1 represent the residual projectile velocity after complet-

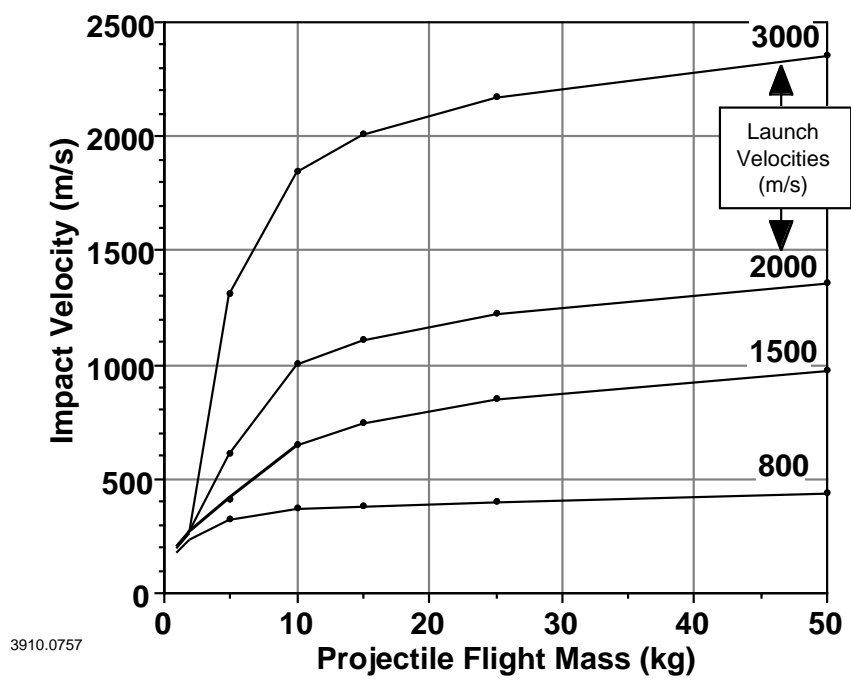

Fig. 1. Higher launch velocities enable high velocity kinetic kill at impact.

ing a ballistic trajectory, although the range of the projectile also varies significantly with launch velocity as will be discussed later. Conventional guns today, with muzzle velocities of $800 \mathrm{~m} / \mathrm{s}$, cannot achieve sufficient velocity at range for kinetic energy lethality.

The use of higher velocity, high density projectiles brings with it greatly increased range. Addition of either chemical explosives or rocket propellants lowers the average density and increases the size and drag of the flight body. In Fig. 2, the ballistic range of conical kinetic energy projectiles launched at velocities between 0.8 and $3 \mathrm{~km} / \mathrm{s}$ is plotted. As density is decreased (for projectiles carrying explosives), the diameter of the flight body, and therefore the aerodynamic drag, increases rapidly. For small kinetic energy projectiles, launch at high velocity allows them to exit the dense atmosphere quickly, and then be directed toward the target using aerodynamic control surfaces with relatively little drag. High flight Mach numbers also leads to substantial reductions in aerodynamic drag coefficients as shown in Fig. 3. Higher launch velocities enable higher impact velocities as shown previously in Fig. 1. If the flight velocity to impact remains above about Mach 3, a larger fraction of the launch kinetic energy can be retained to the target. This kinetic energy efficiency effect is illustrated in Fig. 4. Fig. 4 shows that launch at $3,000 \mathrm{~m} / \mathrm{s}$ leads to much higher efficiency if the projectile is large enough so that its velocity remains above the high drag region below Mach 3 all the way to impact.

In order to provide significantly greater lethality than current $155 \mathrm{~mm}$ rounds, system designs for guns capable of launching 5 to $10 \mathrm{~kg}$ guided kinetic energy projectiles at up to $3 \mathrm{~km} / \mathrm{s}$ were investigated. A notional concept for this type of 


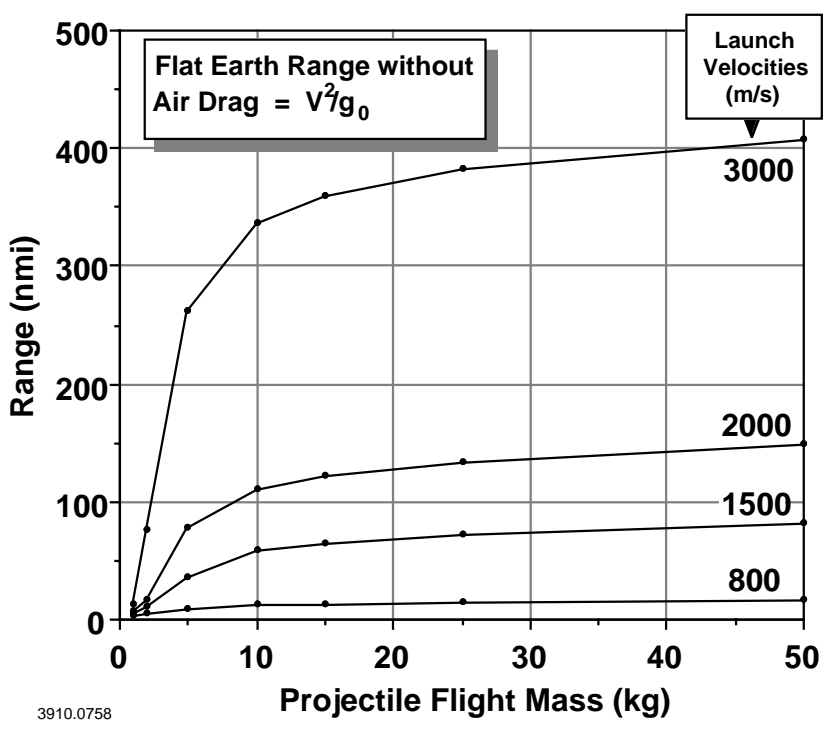

Fig. 2. Higher launch velocities and high density low drag projectiles enable long ranges even with modest projectile sizes.

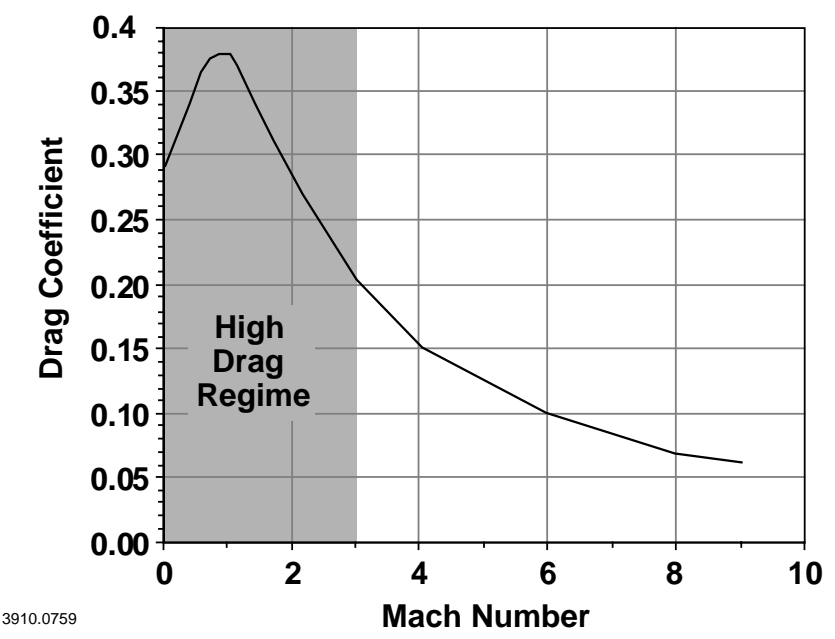

Fig. 3. Drag coefficients increase to high levels below Mach 3.

projectile is shown in Fig. 5, and uses a conical tungsten flight body which contains an integral guidance package and moveable surfaces for aerodynamic flight control. A base-pushing solid armature provides the acceleration mechanism and rear stabilization of the round while in-bore. The armature and armature support structural element are aerodynamically discarded after muzzle exit. A forward bore-rider, a multi-piece structure, is also used for in-bore stability and likewise is discarded upon muzzle exit. Preliminary studies indicate that the flight projectile will be approximately $50 \%$ of the overall launch mass.

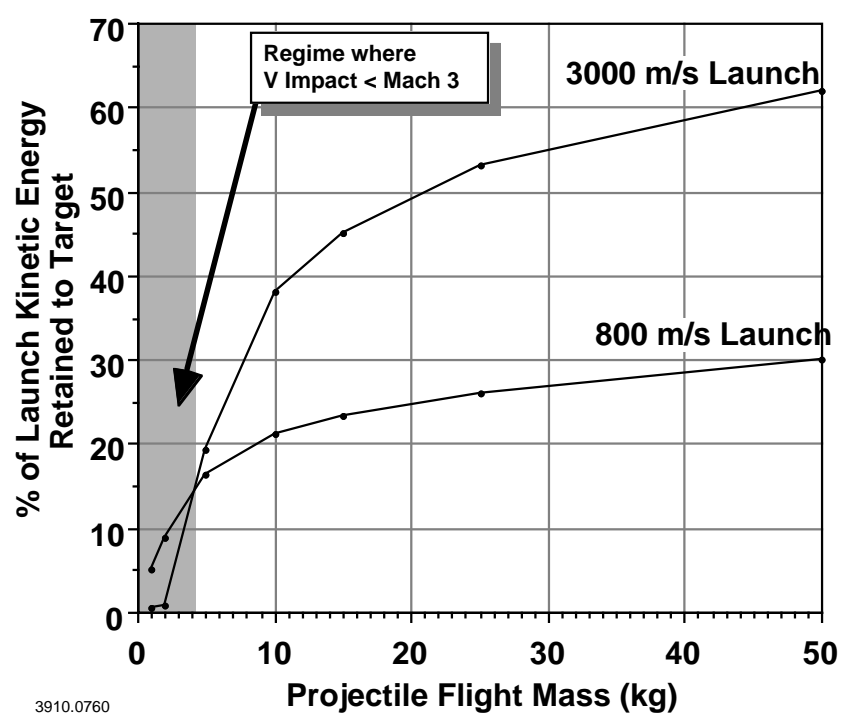

Fig. 4. Higher launch velocities lead to higher efficiency for projectile masses large enough to maintain velocities above Mach 3 throughout the flight.

Sizing of the railgun bore depends on three key parameters: allowable effective bore pressure $\left(\mathrm{P}_{\mathrm{b}}\right)$, piezometric launch efficiency (Z), and barrel length (L). Railgun effective bore pressure is defined as:

$$
P_{b}=\frac{\text { Electromagnetic Accelerating Force }}{\text { Railgun Bore Area }}=\frac{\frac{1}{2} L \mathbb{G}^{2}}{\frac{\pi}{4} D_{b}^{2}}
$$

where

$$
\begin{aligned}
L^{\prime} & =\text { gun inductance gradient } \\
I & =\text { gun current } \\
D_{b} & =\text { bore diameter }
\end{aligned}
$$

and allowable operating values largely depend on the structural characteristics of the barrel. Previous railguns [3] [4] [5] have been successfully operated in the 350 to $450 \mathrm{MPa}$ regime. For this study, operation at $480 \mathrm{MPa}$ is assumed based on improved structural designs which use high strength copper alloy rails, stiff sidewall insulators, and a combination of metal laminates and composite materials in the structure. Piezometric launch efficiency is more a function of the power supply used to drive the railgun. At this writing, piezometric efficiencies of about $50 \%$ have been demonstrated using large laboratory power supplies, and the use of compensated pulsed alternators is being explored to approach $70 \%$ with more compact power sources. Finally, barrel length for these applications is anticipated to be in the 10 to $12 \mathrm{~m}$ range. Railgun bore sizes for these conceptual projectiles are shown in Fig. 6 for assumed system parameters. 


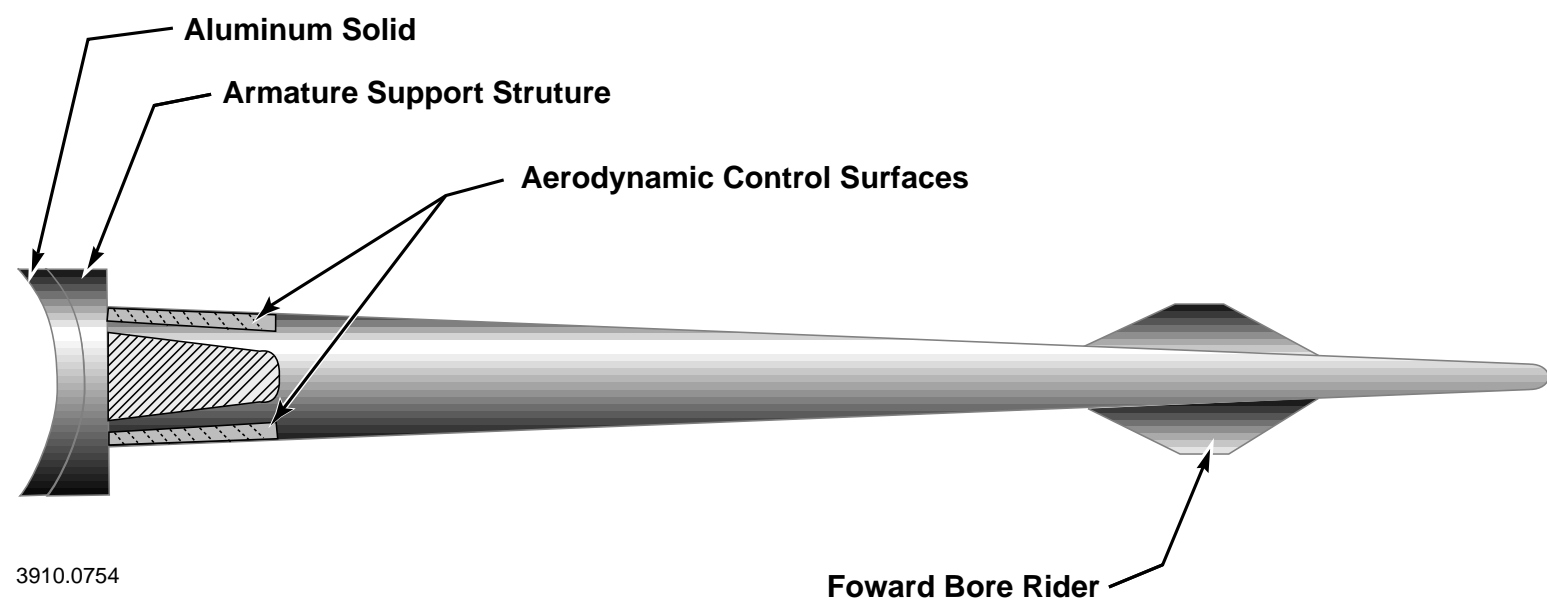

Fig. 5. Preliminary EM railgun launch package concept for NFS applications.

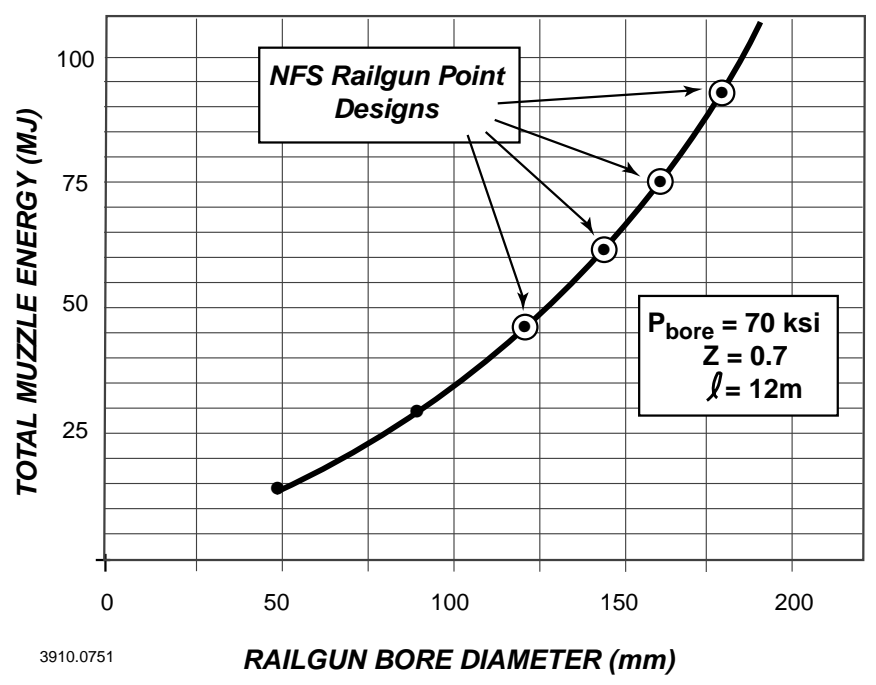

Fig. 6. Railgun muzzle kinetic energy as a function of bore size, peak bore pressure, piezometric efficiency and barrel length.

The pulsed power supply technology required for the NFS concepts is currently under development in the U.S. Army program. Compensated pulsed alternators (compulsators) were invented [16] at The University of Texas Center for Electromechanics in 1978 as a power source for laser flashlamps. A self-excited, air-core variant of the original machine which uses a high speed composite rotor to both store rotational kinetic energy and produce the required power for the railgun launch is envisioned for the NFS gun system.
A likely configuration of the compulsator pulsed power supply is a pair of counter-rotating machines which are parallel connected to the railgun through appropriate switches. While this approach requires that the two machines be synchronized, it provides the benefits of both gyroscopic and discharge torque cancellation to the vessel platform. The projected size of the compulsator power supply is shown in Fig. 7 for a range of projectile energies. This pulsed power system size can easily be accommodated within the volume available in an existing U.S. Navy 5 in. gun mount.

Prime power requirements for the NFS railgun depend on firing rate, launch energy, and overall system efficiency. It is conceivable to have very high burst firing rates (limited only by projectile loading times) for a few shots using the stored energy in the compulsator rotors. The number of launches possible at this rate is governed by the amount of kinetic energy stored in the rotor(s) and thermal considerations. From a practical compulsator design standpoint, however, the number of stored shots will likely be less than six. Since railgun efficiencies improve with higher velocity, overall system efficiencies (rotor energy to launch energy) of 35 to $45 \%$ are feasible using near-term compulsators and railguns.

\section{INTEGRATEd ELECTRIC SHIP SyNERGISM}

In the development of an integrated electric power system for future naval vessels, there is a need for an intermittent energy storage function to help in the stabilization of the electric power distribution system. Technologies of interest for this energy storage device include superconducting magnetic energy storage (SMES), electrochemical batteries, and flywheels. The pulsed power system needed to drive either the fire support or the ship defense railgun as described above would be a pair of counter-rotating compulsators storing several hundred MJ. The interface with the power distribution 


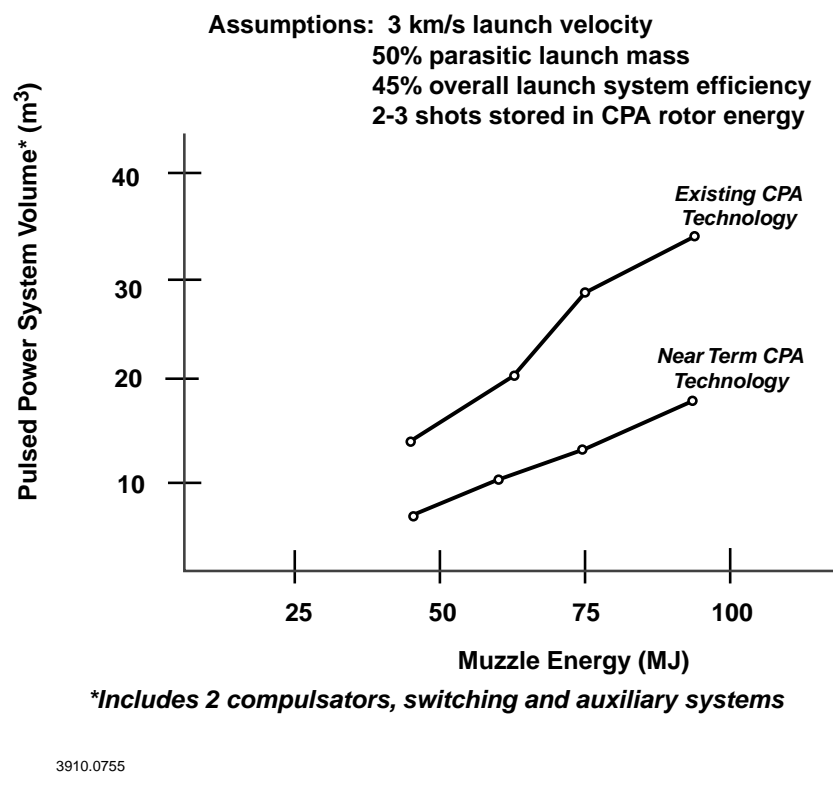

Fig. 7. NFS railgun pulsed power supply size estimates. tems have been developed and current Navy programs are pursuing hardware demonstrations of these devices. Some additional development of certain guidance system components, such as the clock crystal, may also be needed to allow them to sustain the accelerations expected in a high velocity railgun launch. Second, development of aerodynamic control systems needed to steer the projectile at very high velocity is necessary. This approach is being developed for lower velocity systems, but their operation at near Mach 10 has not been addressed. With regard to the railgun barrel, manufacturability and bore life issues must be addressed. Railgun barrels, unlike conventional gun tubes, are multi-piece structures which must be assembled and operated without compromising bore straightness or dimension. Damage to the bore materials (rails and sidewall insulators) of the gun must also be avoided, and more fundamental research is needed to develop a long bore life gun tube. Finally, the most significant challenges facing the compulsator pulsed power supply are the switching systems required and the thermal management of the high speed rotor. Near term switching systems employing vacuum arc switches will likely be adequate for naval applications, although the emergence of $\mathrm{SiC}$ solid state devices may provide a more compact switch with reduced losses in the future.

\section{SUMMARY}

system would be an electric motor used to spin up the compulsator rotor. By operating the motor as a generator, the energy stored in the compulsator rotors can also be accessed for other uses, including distribution system stabilization and emergency power.

A likely operational scenario in an electric ship involves the use of multiple turbine driven generators to provide power generation for the electric drive and other ship functions. The use of several smaller units allows only the number of turboalternators needed to meet the immediate ship power requirement to be operating. This can help significantly in overall efficiency by allowing each of the turbines to operate at full capacity, and thereby reducing the time they operate at less efficient partial power states. Another advantage of multiple power generation modules is that they can be distributed around the vessel to improve survivability if desired. The difficulty arises when power demand increases rapidly because of the time required to bring an additional turbo-alternator online. The compulsator rotors, acting as flywheels, can supply the increased demand for the start-up period required by the turbine. Also, they can be used to absorb excess power from the distribution system when large electrical loads are abruptly disconnected.

\section{Technology Challenges}

While the application of EM guns and guided projectiles to naval missions provides a significant level of attractiveness, there are certain technology challenges which must be addressed. First, miniaturization of the control guidance electronics package is required. Concepts for these types of sys-
Over the past 15 years, railgun and guided projectile technologies have evolved significantly and can now be realistically evaluated for naval applications. Preliminary investigations into fire support missions show that high velocity, high density projectiles can easily achieve ranges beyond $100 \mathrm{~nm}$, and may be able to operate at up to $400 \mathrm{~nm}$. Pulsed power supply, railgun barrel and projectile designs for both applications can be postulated based on existing hardware, or hardware currently under development. Size and mass of these systems appears to be well within reason for integration into naval vessels, and prime power requirements are compatible with installed power being contemplated for future ships with integrated electric power systems. Furthermore, the railgun power supply not only integrates well into a future electric ship architecture, it can serve the additional functions of power distribution system stabilization and a source of emergency power.

\section{REFERENCES}

[1] J.H. Price, E.P. Fahrenthold, D.R. Peterson, W.F. Weldon, R.C. Zowarka, W.G.C. Fulcher, and M.W. Ingram, "Design and testing of large-bore, ultra-stiff railguns," IEEE Transactions on Magnetics, vol. 25, no. 1, January 1989.

[2] J.D. Powell, "Plasma analysis of a large-bore, arc-driven railgun," IEEE Transactions on Magnetics, January 1989, vol. 25, no. 1, pp. 448-453.

[3] R.L. Laughlin, J.L. Bacon, R.C. Zowarka, and J.H. Price, "Design, analysis, and fabrication of two lightweight, high L' railguns," IEEE Transactions on Magnetics, vol. 29, no. 1, January 1993. 
[4] J.D. Herbst, "Installation and testing of a high L' laminated railgun," IEEE Transactions on Magnetics, vol. 33, no 1, January 1997.

[5] A.E. Zielinski, M.D. Werst, and J.R. Kitzmiller, "Rapid fire for the cannon caliber electromagnetic gun system," IEEE Transactions on Magnetics, vol. 33, no 1, January 1997.

[6] R.L. Laughlin, J.H. Gully, K.E. Nalty, and R.C. Zowarka, "System design of the ultra-high GEDI experiment," IEEE Transactions on Magnetics, vol. mag-22, no. 6, pp. 15781583, November 1986.

[7] D.A. Weeks, W.F. Weldon, and R.C. Zowarka, "Plasma armature launcher simulations at The University of Texas at Austin," IEEE Transactions on Magnetics, vol. 25, no. 1, January 1989.

[8] R.L. Fuller, J.R. Kitzmiller, and M.W. Ingram, "Design and testing of a 0.60 caliber augmented railgun," IEEE Transactions on Magnetics, vol. 27, no. 1, January 1991.

[9] J.H. Price and H.D. Yun, "Design and testing of integrated metal armature sabots for launch of armor penetrating projectiles from electric guns," IEEE Transactions on Magnetics, vol. 31, no. 1, January 1995.

[10] J.H. Price, H.D. Yun, K.G. Cook, J.P. Kajs, H.P. Liu, and M.D. Werst, "Discarding armature and barrel optimization for a cannon caliber electromagnetic launcher system," IEEE Transactions on Magnetics, vol. 31, no. 1, January 1995.

[11] J.R. Kitzmiller, S.B. Pratap, T.A. Aanstoos, K.G. Cook, R.A. Kuenast, B.T. Murphy, and D.R. Perkins, "Optimization and critical design issues of the air core compulsator for the cannon caliber electromagnetic launcher system (CCEML)," IEEE Transactions on Magnetics, vol. 31, no. 1, January 1995.

[12] J.R. Kitzmiller, S.B. Pratap, M.D. Werst, C.E. Penney, T.J. Hotz, and B.T. Murphy, "Laboratory testing of the pulse power system for the cannon caliber electromagnetic gun systems (CCEMG)," IEEE Transactions on Magnetics, vol. 33, no. 1, Jan 1997.

[13] B.D. Goodell, J.S. Perry, M.V. Atkinson, E.B. Goldman, J.T. DeWitt, T. Mohler, and J.R. Kitzmiller, "Electromagnetic (EM) weapon system integration into combat vehicles," IEEE Transactions on Magnetics, vol. 32, no. 1, Jan. 1995.

[14] S. Fish and E. Redding, "Prime Power and Pulsed Energy Storage for EM Gun Equipped Tank Combat Missions," IEEE Transactions on Magnetics, vol. 33, no. 1, Jan. 1997, pp. 642646.

[15] U.S. Navy 1997 Strategic Studies Group Final Report.

[16] U.S. Patent No. 4, 200, 831, April 29, 1980, W.F. Weldon, M.D. Driga, and H.H. Woodson. 